\title{
EL DESTINO COMÚN DE LA ECONOMÍA Y LA TEORÍA: DE SU ORIGEN Y SU EPÍLOGO.
}

\section{ALEJANDRO ROJAS JIMÉNEZ*, Universidad de Málaga}

Resumen: En este trabajo se comenta el paso del Neolítico a las civilizaciones antiguas rastreando la relación esencial entre el nacimiento de la economía de producción y el origen de las ciencias, vinculando finalmente la crisis de las ciencias europeas con la crisis de la economía cuando, devaluada la actitud emancipadora propia de la economía de producción, la teoría queda desasistida de su sentido real que es liberar del mundo de medios pensando más allá de lo posible.

teoría, crisis, economía, origen,

Revolución neolítica, civilizaciones antiguas

Sumary: this paper discusses the transition from the Neolithic to the ancient civilizations tracking the essential relationship between the birth of Economies of Production and the origin of sciences, linking the Crisis of European Sciences with the economic crisis when, the emancipator attitude typical of Production Economics is devaluated, the theory is neglected in its real sense which is to free the world of means thinking beyond of what is possible

theory, crisis, economy, origin,

Neolithic Revolution, ancient civilizations

\section{DEL NEOLÍTICO A LAS CIVILIZACIONES ANTIGUAS}

a) De la economía de supervivencia a la economía de producción

La prehistoria de la economía de producción es el Neolítico y el inicio de la vida sedentaria asociado a la domesticación y la agricultura. Este período que marca el fin del Paleolítico, es tan importante que bien ha merecido el calificativo de Revolución Neolítica. Los especialistas entienden que los cambios climáticos producidos, así como la red de utensilios $^{1}$ disponibles, abrieron la posibilidad de la agricultura y la vida sedentaria propias del Neolítico. Menos atención por su parte se ha puesto para entender el paso que va desde el Neolítico a las primeras civilizaciones

\footnotetext{
*rojas_a@uma.es

1 „Damit aber das Dasein mit einem Zeugzusammenhang soll umgehen können, muß es so etwas wie Bewandtnis, wenngleich unthematisch, verstehen: es muß ihm eine Welt erschlossen sein “ $M$. Heidegger Sein und Zeit (GA 2) §69 b
} 
donde la economía de producción ${ }^{2}$ sustituyó definitivamente a la economía de supervivencia: en el Antiguo Egipto y Mesopotamia producían trigo, en la India y China arroz, y en América central maíz. Esta desatención seguramente se debe a que existe la tentación de considerarlas como una evolución lógica del Neolítico, pero esto olvida lo que desde mi punto de vista es fundamental: que el paso a una auténtica economía de producción que sustituye definitivamente a una economía de supervivencia (cosa que no acaba de ocurrir en el Neolítico) se produce cuando los factores que permitieron el paso del Paleolítico al Neolítico ya no sirven como explicación, en tanto que incluso son un impedimento.

Quiero decir, mientras que fueron factores externos los que explicaron la Revolución Neolítica, especialmente el cambio climático al permitir la aparición de la agricultura, sin embargo, la Revolución Histórica que supone el paso del Neolítico a las civilizaciones antiguas se produce cuando los factores externos no son suficientes para sostener el incremento de la población y propagación de la vida que había favorecido la Revolución Neolítica. Un vuelco de la situación que entiendo de esta manera: si en el Neolítico el cambio climático abre las puertas a un mundo de nuevas posibilidades, estas nuevas posibilidades abiertas por el cambio climático despertaron en los hombres el goce de vivir al acecho de posibilidades futuras, e impulsados por esta nueva forma de vivir el Neolítico se convierte en el inicio del fin de la prehistoria en la medida en que una vez que los hombres empezaron a soñar con futuras posibilidades, sus proyectos y expectativas fueron más allá de lo que el cambio climático hacía posible (el fin del Neolítico), hacia posibilidades que son por primera vez en la historia auténticamente futuras.

El hombre aprendió a soñar lo imposible, y creyó en su capacidad para hacer posible lo todavía imposible. Los útiles de hueso que se elaboraron en el Paleolítico superior (como el propulsor o el arco) servían para cazar, pero a partir del Neolítico la caza no tendrá como fin únicamente alimentar a la población, sino alimentar a la población futura, porque el hombre había decidido y creído posible crecer más allá de lo que su mundo de medios y su entorno hacía posible. Y para este propósito valía todo, incluso multiplicar las divinidades (como parece que ocurrió durante el Neolítico y la Edad de Bronce). Como consecuencia de esta nueva disposición se produce la sustitución de la economía de supervivencia por la economía de producción. Porque el mundo de medios de los que disponen los hombres no garantiza el mantenimiento y la propagación de la vida que en el Neolítico se había generado, y que el mismo Neolítico no pudo contener. Ni siquiera la Edad de los metales, en los que la humanidad incrementó sus posibilidades pudo contener sus ansias de futuro. Cuando apareció el modo de abrirse a lo imposible, más allá de lo que el mundo de medios hace posible (más allá incluso que los metales), la humanidad empieza a vivir el inicio de su historia y el fin de la prehistoria. Una sustitución

\footnotetext{
2 "ha tenido vigencia desde el Antiguo impero Egipcio hasta la caída del Imperio Romano, y después del paréntesis medieval, desde el comienzo del estado moderno hasta la Primera Revolución Industrial" J. Choza, Antropología filosófica. Representaciones del sí mismo (Biblioteca Nueva, Madrid, 2002) p 168
} 
marcada por la siguiente actitud diferencial: ya no se trataba de sobrevivir (que es vivir en la medida de lo posible) sino de producir lo necesario para sostener una vida que viva proyectada hacia un futuro que excede lo posible. Este modo de abrirse a lo imposible, que nace vinculado a la economía de producción y que a mi juicio es la nota fundamental de la historia frente a la prehistoria de la humanidad, es la teoría, la ciencia.

Si pensamos que el paso del Neolítico a las civilizaciones Antiguas es sencillamente una suerte de evolución progresiva, entonces quedaremos asombrados y estupefactos ante la grandiosidad de las edificaciones de las civilizaciones antiguas, sobretodo en comparación con la prehistoria de la humanidad. Desde dicho asombro se construyen historias exóticas para explicar cómo unos hombres con tan pocos medios pudieron hacer aquellas colosales estructuras así como inventar la escritura o las matemáticas. Frente a estas posiciones, debe observarse que fue precisamente la liberación de su mundo de medios, lo que les llevó a vivir por encima de las posibilidades abiertas por aquel.

Y como respuesta a aquellos que se asombran de la cantidad de tiempo que fue necesario para que la humanidad emprendiera dicha liberación de su mundo circundante, hay que decir que desde luego es mucho más sencillo soñar sólo con la posibilidad efectiva, que es lo que se dice coloquialmente "vivir con los pies en la tierra". Era necesario que ocurriera que los proyectos que no son posibles apremiaran hasta el punto de ser irrevocables, para que entonces aquella existencia apacible cediera al vértigo del futuro; al vértigo de la aventura por abrirse más allá de las posibilidades dadas. Y esto significa ceder al vértigo de la teoría, porque es la teoría la práctica intelectual que se abre más allá de las posibilidades dadas. Es interesante resaltar cómo la civilización actual ha sido absorbida por el gran poder de los medios que lejos de presentarse como finitos y limitados, resultan en su asociación con la ciencia (asociación a la que damos el nombre de tecnología) enormemente poderosos. Nuestra civilización piensa que es ilegítimo pensar por encima de nuestras posibilidades, pensar más allá de lo posible. Obras básicas de nuestra civilización son por ejemplo la crítica de la razón pura y el discurso del método. Y es que si lo posible se antojaba en el Neolítico demasiado poco, hoy podemos mucho más de lo suficiente. Pero el problema no era de poder, sino de libertad. Y la libertad, frente a lo que pensaba Nietzsche no tenía que ver con el poder, ni el querer, sino con el pensar. La liberación del pensar es el paso del Neolítico a las civilizaciones antiguas, que hoy vuelve a estar en peligro bajo el yugo de la voluntad de poder que favorecen los medios. Pues no se trata de hacer lo que se nos ocurra, sino de pensar más allá de lo que se puede hacer. Tal es la libertad respecto del mundo. 


\section{b) ¿Existencia libre o subordinada a lo posible?}

El hombre puede pensar su mundo. Hoy en día parece que ésta es además la tarea de las ciencias, conocer el mundo, e incluso que no puede pensarse otra cosa que el mundo. El rechazo a saberes como la metafísica, cuyo tema no es el mundo, sino (después de considerar la posesión del objeto por el pensamiento en su independencia respecto del fundamento) su causa real más allá de la objetividad, tiene su base sobre esta consideración de que lo que debe hacer el hombre es conocer su mundo. Pero no es cierto que el hombre sólo pueda conocer su mundo, de hecho, y quiero poner énfasis en esta tesis, el intelecto puede abrirse a otros mundos posibles.

Se suele decir que esto es "fantasear", y no es falso que haya más filosofía en la literatura que en la historia de hechos ${ }^{3}$, pero no es exactamente lo mismo pensar futuras posibilidades, que pensar unicornios. Los unicornios no son futuros, sino un presente imaginario. Y no se trata de pensar otros presentes distintos, sino futuros mundos: un mundo, por ejemplo, en el que se pueda transportar mercancía por el Nilo en sintonía con el pensamiento de un mundo en el que se aprovechara al máximo la crecida del Nilo. Obviamente el máximo no es alcanzable, pero en pos de este futuro tendrán que abandonar el presente. Sólo un pueblo abierto al futuro de esta manera puede hacer barcos. Ciertamente se podría explicar las construcciones de las primeras embarcaciones en función de los medios, y autores como Heidegger ya han avisado de que la red de renitencias de los útiles del mundo nos anticipa la funcionalidad del útil ${ }^{4}$, pero incluso el propio Heidegger acabará por conceder con el tiempo que pensar el futuro no tiene que ver con comprender nuevas posibilidades abierta por el mundo de medios, sino con la capacidad de penar más allá de lo que el mundo de medios hace posible, y por eso, dirá que un pensar así es un pensar abierto al misterio ${ }^{5}$. Y es que un mundo en el que se pueda transportar mercancía por el Nilo no ocurre porque los medios lo hicieran posible, sino porque la humanidad tenía una tarea urgente que resolver, que le demandaba a abrirse a lo misterioso más allá de sus posibilidades.

Heidegger había hablado de dos tipos fundamentales de existencia:

\footnotetext{
3 "Y es evidente también a partir de lo dicho que la función del poeta no es contar lo sucedido, sino lo que podría suceder y lo posible en virtud de la verosimilitud o la necesidad. Pues el historiador y el poeta no difieren entre sí por escribir en prosa o en verso, ya que podrían versificarse las obras de Hesíodo y no serían en absoluto menos historia con verso que sin verso. La diferencia estriba en que uno narra lo sucedido y el otro cosas tales que podrían suceder. Por lo cual precisamente la poesía es más filosófica y seria que la historia, pues la poesía narra más bien lo general, la historia lo particular" Aristóteles, Poética 1451 a36-b5

${ }^{4}$ „Vorgänging »sein« Lassen besagt nicht, etwas zuvor erst in sein Sein bringen und herstellen, sondern je schon »Seiendes « in seiner Zuandenheit entdecken und so das Seiende dieses Seins begegnen lassen. Dieses apriorische Bewendenlassen ist die Bedingung der Möglichkeit dafür, da $\beta$ Zuhandes begegnet, so da $\beta$ das Dasein in ontischen Umgang mit so begegnenden Seinden, es im ontischen Sinne dabei bewenden lassen kann" M. Heidegger, Sein und Zeit cit § 18

${ }^{5}$ „Die Offenheit für das Geheimnis“ M. HEIDEGGER, Gelassenheit (Neske, Pfullingen, 1959), p 24
} 
a) La primera, denominada por Heidegger como impropia ${ }^{6}$, por quedarse rezagada "en casa", donde el hombre no se deja arrastrar por sus sueños, y vive satisfecho en aquello que su mundo hace posible. (Confieso que pueden haber teorías para justificar una actitud semejante como las estoicas o las epicúreas: recuerden a Diógenes diciéndole al Gran Alejandro que se aparte del Sol), pero observen que estas posiciones se dieron precisamente cuando el yugo del imperio impedía el pensamiento libre: deben ser entendidas como la justificación de una renuncia a la libertad de pensar, como consecuencia de una pérdida político-social de libertad que obliga a una sumisión de los sueños a las posibilidades dadas.

En este caso la teoría (el pensamiento que vuela más allá de lo posible ${ }^{8}$ ) muere por miedo: no se está dispuesto a luchar por hacer posible los sueños en aquellos casos en que dichos sueños no son posibles en una situación dada.

b) Pero por otro lado, cabe también llevar una existencia auténtica, mantengo la terminología heideggeriana: vivir liberándose de su mundo de medios (algo que en última instancia hace apaciguando la voluntad de poder, y renunciando a querer $\left.{ }^{9}\right)$, y esto significa pensando más allá de lo que se puede: meditando serenamente. Una meditación que en la filosofía del segundo Heidegger sustituye a la comprensión, cumpliéndose la sustitución de la proyección de posibilidades propias por la escucha serena, pero que mantiene la misma intención: la apertura de nuevos caminos por recorrer hacia nuevos lugares ${ }^{10}$ futuros.

Hay que decir que desde el pensar meditativo el futuro se piensa como Destino en el que descansa la historia del pensar (Destino como esencia de la verdad), pero no quiero profundizar en este asunto. Sino en este otro: el futuro que se asocia a la existencia auténtica no es un futuro presente por venir (sólo la muerte es un futuro presente por venir), sino un futuro que se proyecta como tarea que nos mueve y abre caminos por recorrer. De modo que proyectar la posibilidad de transportar mercancía por el Nilo no es un futuro posible que se haga presente al pensar, pongamos por caso, una canoa, sino una tarea que va más allá de las canoas y en pos da la cual se despliega toda una historia de las embarcaciones.

\footnotetext{
6 "In dieser Vertrautheit kann sich das Dasein an das innerweltlich Begegnende verlieren und von ihm benommen sein" M. Heidegger, Sein und Zeit cit $\$ 17$

7 "«existir», «existencia», significa en realidad «estar fuera de». Cuando Heidegger en Ser y Tiempo dice que «ser-en», «estar-en», significa «habitar», a donde en realidad nos lleva es a otro significado de habitar. La única habitación, fuera de la caverna, en la existencia, es el mundo y no una construcción arquitectónica que nos proteja del exterior" A. Leyte, Figuras constructivas del paisaje, en Sileno. Variaciones sobre arte y pensamiento 11(2001) 8-17 p 12

8 „Die leichte Taube, indem sie im freien Fluge die Luft theilt, deren Widerstand sie fühlt, könnte die Vorstellung fassen, daß es ihr im luftleeren Raum noch viel besser gelingen werde" KRV B8-9

${ }^{9}$ Frente a Nietzsche, la Gelassenheit

${ }^{10}$ „Der Weg ist, hinreichend gedacht, solches, was uns gelangen läßt, und zwar in das, was nach uns langt, indem es uns belangt" M Heidegger, Das Wesen der Sprache (GA 12) p 186
} 
Pues bien, llegados a este punto creo que se puede exponer con claridad el modo decisivo en el que está vinculado el origen de la economía y el origen de las ciencias.

\section{EL ORIGEN DE LA ECONOMÍA Y EL ORIGEN DE LAS CIENCIAS}

Del mismo modo que Romeo y Julieta podrían servirnos como modelos para defender una existencia que, proyectando lo imposible, no renunciara a sus sueños, el Antiguo Egipto puede servir para mostrar claramente cómo los medios de los que un pueblo dispone pueden llegar a ser escasos para saciar las expectativas de futuro de sus gentes. No estoy diciendo que de pronto los medios fueran escasos. La escasez es sólo escasez debido a los proyectos humanos: la escasez la introduce el hombre cuando sueña más allá de las posibilidades dadas ${ }^{11}$.

En el Antiguo Egipto la vida proyectada era posible sólo por las rentas de métodos realmente eficaces para aprovechar la credida del Nilo. La idea de continuar con la propagación de la vida que favoreció el Neolítico se enfrentaba con el problema de que empezaba a ser insuficiente simplemente buscar un método eficaz; en el Antiguo Egipto sólo valía la máxima eficacia.

Cuando no se trata de la búsqueda de lo más eficaz, sino tan sólo de buscar una solución eficaz, ésta no tiene porqué obedecer a una elección sabia, el hombre por ejemplo puede elegir pasionalmente su camino o entender que es mucho más eficaz una decisión consensuada que la mejor elección. Aunque la elección racional ha gozado siempre de muy buena prensa (por otro lado justificada) considero que la elección pasional es mucho más hermosa y tan humana como aquella; incluso mucho más común entre los hombres. Es muy humano dejarse llevar por las pasiones, que para Aristóteles eran: la concupiscencia, la cólera, la audacia, el temor, la envidia, el gozo, el amor, el odio, el pesar, los celos y la compasión. Nótese qué bien reflejaban los mitos griegos la importancia de todas estas pasiones en la vida del hombre. Tan importante eran, y tan humano verse afectado por ellas, que los clásicos tuvieron el sabio gesto de divinizarlas ${ }^{12}$. ¡Qué humano es y que respetable, ante el problema de la escasez, elegir inspirado por la Audacia (representada por Atenea)!

Pero cuando se trata de la búsqueda por la mayor eficacia, la pasión, la autoridad o el parloteo no valen. Si la humanidad dejó de plantearse como fin hallar una respuesta eficaz para solucionar un problema concreto, y ante la urgencia de aprovechar al máximo la crecida del Nilo, la humanidad se preguntó ¿cómo se puede aprovechar más la crecida del Nilo? en este momento introdujo una gran novedad:

\footnotetext{
11 “el mundo no es escaso, más bien es todo lo contrario, rico y abundante; la escasez la introduce el hombre al abrir y crear posibilidades que no existían en el mundo" I. F. Sorauren, El fin mínimo de la actividad económica, en Urquía-Ureña-Muñoz (eds), Estudios de teoría económica y antropología (Unión editorial, Madrid, 2005) 647-685, p 671

${ }^{12}$ Cfr, W. F. OTTO, Los dioses de Grecia. La imagen de lo divino a la luz del espíritu griego (traducción de Rodolfo Berge y Adolfo Murguía, Eudeba editorial, Buenos Aires, 1973)
} 
una pregunta cuya respuesta es imposible, no por desconocimiento, sino porque en sus mismos términos indica ya la necesidad de superar lo encontrado. Una pregunta así, que despegaba la cabeza de lo concreto (hábito abstractivo: lenguaje), se hizo en primer lugar generalizando, porque la generalización permitía abandonar el terreno de lo concreto: no se trataba de esta o aquella crecida, sino de las crecidas en general. La escritura, la geometría y las matemáticas nacen asociadas a esta búsqueda por el método más eficaz de sacarle más partido a las crecidas del Nilo en general.

Así demandados por esta tarea, los hombres son arrastrados a una persecución incesante que es el auténtico inicio de la historia de la humanidad: una historia de descubrimientos continuamente abandonados por no remitir a un descubrimiento concreto.

Pongamos el siguiente ejemplo imaginario: dos cazadores que están ante un ciervo con una gran cornamenta. Deben cazar la pieza para poder sobrevivir, pero no pueden acercarse sin más a cogerla. De modo que se ponen a discutir acerca de cómo hacer efectiva dicha tarea. Al hablarlo se cuentan cómo otros hombres lo habían hecho antes que ellos, recuerdan la leyenda sobre el héroe que cazó a tres ciervos con una sola piedra o cómo después de la muerte todo está lleno de ciervos. Incluso hablarán acerca de cómo innovar en la práctica de la caza del ciervo sirviéndose de propias experiencias. Pues bien, lo que debe ser destacado de esta práctica es que en el momento del éxito práctico acabó la reflexión.

Imaginaros ahora a esos mismos individuos, intentando decidir la mejor forma de cazar a un ciervo. Ante una pregunta semejante, debe notase que poco importa el ciervo al que habían ido a dar caza. El universo teórico se sobrepondría sobre el empírico, y seguramente volverían a casa con un montón de ideas, la mayoría todavía por someterlas a una reflexión más exhaustiva... pero sin ciervo concreto, porque siempre estuvieron hablando de los ciervos en general (los ciervos los traían a montones, pero en la cabeza). Sus mujeres les reñirían, sus hijos los odiarían, y sus amigos se mofarían de ellos; sobre todo cuando el anciano de la ciudad les hiciera ver haciendo del caso escarnio público: que la mejor manera de matar a un ciervo concreto era dándole muerte concreta.

Pero, y si de repente las circunstancias cambiaran y fuera necesaria la labor de estos locos teóricos. Esto es lo que pasó en el Antiguo Egipto. Los sueños y expectativas de éstos les obligaba a aprovechar al máximo los recursos y el tiempo. Lo que invalidará la decisión del anciano (con más experiencia), la decisión del fuerte, y la decisión del grupo mayoritario, incluso la del héroe... es que ya no se tratará sólo de responder eficazmente, sino de no dejar de preguntarse de qué modo lograr la mayor eficacia para aprovechar los recursos de cara a los proyectos del grupo. Ya no se trataba de sobrevivir, sino de sostener los sueños, y por eso había que hacer algo más que aprovechar los recursos, había que producir lo necesario en pos de un sueño. 
No me cabe duda de que el hombre también hubiera sobrevivido si hubiera sido más modesto en sus proyectos, pero impulsados por el deseo de procrearse y de extenderse, o les sacaban más partido al desbordamiento del río africano, o cerraban los ojos a sus sueños y expectativas de futuro que habían sido favorecidas en el Neolítico. En este momento, la tarea ya no tenía que ver con hallar una respuesta concreta, sino que exigía una reflexión constante y no desfuturizable acerca de cómo sacar más partido a las crecidas del Nilo en general (se perseguía ese más a la respuesta).

En el Antiguo Egipto el hombre aprendió a preguntar más allá de lo concreto. El hombre aprendió en Egipto a no preguntarse ¿cómo transporto esta mercancía por el Nilo?, sino a preguntarse cómo transportar mercancía por el Nilo, en general (historia de las embarcaciones), a no preguntarse como sitúo estas piedras para levantar esta tumba, sino cómo edificar tumbas en general (historia de los enterramientos incluidas las pirámides). Esta y aquella piedra, ceden su lugar a la representación escrita del número de piedras necesarios y su distribución.

La generalización, como continuación de la operación incoativa de la inteligencia ${ }^{13}$, es sólo un primer estadio en el que cabe progresión, una progresión que se dará en Grecia, con Platón y Sócrates, con el reconocimiento de la limitación de la generalización y su abandono a favor del concepto, que es el abandono de la idea general por lo universal. Pero antes de llegar al concepto, desde el estadio de la generalización se abrió todo un abanico de saberes como la escritura o la matemática. $\mathrm{Y}$ aunque si fuéramos rigurosos tendríamos que decir que la historia del pensar debería comenzar propiamente con Aristóteles, e incluso después de éste con el hábito de sabiduría ${ }^{14}$, y que lo que se inició en Egipto era más bien la prehistoria de la teoría, sin embargo, con la generalización comienza la historia de la humanidad y acaba la prehistoria; y esto asociado a una tarea propiamente económica: había que producir lo necesario en pos de un sueño.

Quiero insistir que este futuro no es un futuro presente. Esta manera de abrirse al futuro significa abrirse a lo irrealizable generando historia. Quiero poner el énfasis en que pensar el transporte fluvial no acaba con pensar un barco. No se trata de lo que se hará hoy o mañana, sino de qué es lo que hay que hacer en pos de ese mañana. Como cuando el niño dice que quiere ser un gran bombero. No se llega a ser un gran bombero después de hacer determinadas cosas, sino que se trata de ir haciendo cosas en pos de ese proyecto futuro. Del mismo modo en la infancia de la historia de la humanidad el pueblo egipcio soñó crecer más allá de lo que su mundo hacía posible y se adentró en la inagotable tarea de producir lo necesario. En Antequera tenemos un ejemplo de las primeras manifestaciones de este diálogo

\footnotetext{
${ }^{13}$ L. Polo, Leonardo, Curso de teoría del conocimiento ( tomo II, Eunsa, Pamplona, 2006)

${ }^{14} \mathrm{~J}$. F., Sellés, El conocer más humano y su tema. Sapientia est de divinis según Tomás de Aquino, en Actas del Congreso Internacional Christian Humanism in The Thirt Millenium. The Perspective of Tomas Aquinas, Roma, 21-25 sep. 2003, vol. I, 690-701
} 
existencial con el futuro que apuntaba ya en la dirección de hombres libres que piensan el futuro como algo distinto de lo que está por venir.

\section{DEL PARLOTEO A LA GENERALIZACIÓN}

Antes de la pregunta teórica, durante su prehistoria la humanidad había sido capaz de crear mitos, conjuntos megalíticos, las piezas de orfebrería, los utensilios para pulir, las pinturas rupestres... pero aquí no hay propiamente sabiduría, sino parloteo. Las creencias sobre la vida después de la muerte que da lugar a los dólmenes de Antequera son fruto de un hablar y hablar que bien podríamos denominar charlatanería. El mismo origen tienen las historias míticas y las creencias mágicas. No las descalifico, reconozco que son un modo hermoso de instalar un mundo humano sobre la estéril tierra, pero existe otro tipo de diálogo, muy distinto, que aparece en Egipto provocando el inicio de la historia de la humanidad y el fin de la prehistoria: el diálogo científico. Científico no tiene evidentemente aquí el sentido ordinario de "ciencia moderna", sino que designa el arrebatamiento demónico de la verdad como tarea dialéctica.

El caso es que la mera charlatanería da lugar a saberes gracias a los cuales el hombre se hizo moralmente bueno (los mitos), sobrevivió a su desconocimiento del futuro (magia), incluso avanzó desde la edad de piedra hasta la edad de los metales gracias al habla que les permitía contarse el mundo, contar sus experiencias, los acontecimientos que tenían que ser retenidos, los logros y los fracasos... Pero todas estas historias no pertenecen todavía a la historia de la humanidad, sino a su prehistoria. La reducción actual de la historia a historietas es el recorrido inverso.

El fin de la prehistoria es el fin del agrupamiento para buscar soluciones a problemas concretos. El inicio de la historia es el inicio de la búsqueda científica: la búsqueda emprendida sabiendo de antemano que no hay una respuesta última. Lo que se hizo en primer lugar generalizando. La búsqueda es la práctica que nace del reconocimiento de que lo sabido no sirve iquién mejor que Sócrates supo expresar esto! Fruto de esta sabia búsqueda, que no es sabia por lo sabido, sino por saber que lo sabido no es último, nacen, como hijos suyos, la geometría, la matemática y la escritura y sólo muy posteriormente la filosofía como búsqueda del fundamento real de lo presente. $Y$ todo esto fue posible en la medida en que los hombres se liberaron de su mundo de medios para buscar, en lugar de encontrar, futuras posibilidades hacia las que proyectarse ${ }^{15}$. Siendo así que el surgimiento de la economía de producción es la cuna de las ciencias y de la historia de la humanidad.

De este modo en el Antiguo Egipto se empezó a medir la crecida, a calcular las áreas afectadas por la misma, a escribir las cifras... Bien debemos denominar a la

${ }^{15}$ „Die Entschlossenheit, in der das Dasein auf sich selbst zurückkommt, erschließt die jeweiligen faktischen Möglichkeiten eigentlichen Existierens aus dem Erbe, das sie als geworfenheit übernimmt" M. Heidegger, Sein und Zeit cit, $§ 74$ 
economía como la primera de las ciencias (respetando a la filosofía el ser la ciencia primera) que, insisto, se diferencia de las prehistóricas prácticas de sabiduría en que no se basan en lo sabido y lo dicho, sino en la búsqueda.

\section{LA CRISIS DE LA ECONOMÍA Y LA CRISIS DE LA CIENCIA}

Esta asociación de la actividad teórica esencial a la economía se viene abajo sin embargo cuando la tecnología a la que da lugar la unión entre ciencia moderna y técnica se consolida. En un mundo como el nuestro, donde los medios resultan ser realmente poderosos, dejamos que ellos nos digan la sociedad en la que debemos vivir, sin preguntarnos si la sociedad a la que nos conducen es la sociedad que queremos (Habermas ${ }^{16}$ ).

Una pregunta semejante significaría liberarse de aquellas posibilidades que nos abren los medios, pero la renuncia de la ciencia a este tipo de preguntas y su subordinación a entender el mundo dado inmediatamente a la conciencia (iniciado por Descartes ${ }^{17}$, pero también el empirismo ${ }^{18}$ ) subordina el pensar a la potencia de la inteligencia (derrumbándose saberes como la metafísica o la antropología trascendental que el idealismo alemán intentó salvar). Devaluada la actitud emancipadora propia de la economía de producción, la teoría queda desasistida de su sentido real que es liberar del mundo de medios pensando más allá de lo posible; y se convierte en algo desechable. La primera gran señal crítica de este proceso la vimos en la Revolución Industrial y los problemas sociales que generó: un mundo contrario al que habríamos proyectado, un mundo que manifiestamente nos había sobrevenido y dominado ${ }^{19}$, a pesar de nuestros sueños.

Por eso la tesis marxista fue que la historia tiene sus propias leyes (materialismo histórico). Frente al proyecto de liberación idealista propuso la acción revolucionaria. Y como resulta sin embargo que (ante el fracaso de la tesis marxista) no fue necesaria ninguna revolución para salir airosos, el hombre "aprendió" que de todo se sale: que la propia tecnología nos libraría de los problemas que crea... y en lugar de luchar por la libertad de la humanidad, ésta se creyó segura entre el mundo de útiles. En realidad, no cabe tener miedo a esta crisis económica que repite en menor medida la crisis de la Revolución Industrial. Claro que saldremos... el problema es que cuando salgamos volveremos a querer vivir en la seguridad de nuestros medios. Saldremos de la crisis económica, pero no de la crisis de la economía, que es la crisis de la búsqueda teórica. La economía está al servicio hoy del mercado, pertenece al mundo, y ha dejado de ser esa práctica liberadora que inició la historia de la

\footnotetext{
${ }^{16}$ Cfr., J. Habermas, Ciencia y tecnología como ideología (Tecnos, Madrid, 1984)

${ }^{17}$ R. DESCARTES, Resp. II, AT, VII, p 160

${ }^{18}$ Lo inmediatamente dado a la experiencia (ideas simples)

${ }^{19}$ Marx intentó buscar nuevas formas de liberar a la humanidad, menos teóricas y más prácticas, pero en esta división se ha instalado ya el olvido de que la teoría es la práctica liberadora.
} 
humanidad: el mercado, el consumismo y los servicios ${ }^{20}$ han ahogado a la práctica liberadora que latía en la economía de producción que funcionaba en las civilizaciones antiguas.

Nótese que de ningún modo pretendo decir que se deba volver al Antiguo Egipto o a Mesopotamia, sino que se debe evitar volver al Paleolítico, hacia donde se acerca la historia de la humanidad, y donde la humanidad quedará nuevamente absorbida por su mundo y sin historia. La civilización que es capaz de hacer puentes en lugares imposibles o hacer edificios de 509 metros de altura (edificio Taipei 101), bajo la consideración de que puede hacer cualquier cosa, ha dejado de preocuparse por hacer además de lo que se puede, sobre todo lo que no se puede, que era el sentido emancipador que latía en el origen de la economía de producción y de cuyo impulso nacieron las ciencias.

Puede que hoy vivamos de lo que medios como el petróleo o la electricidad hacen posible, pero en su momento la gasolina (derivado del petróleo) y su uso en los motores de combustión es posterior a la aparición del ferrocarril y un paso más en la historia de estos, del mismo modo que la primera aplicación práctica de la electricidad fue el telégrafo eléctrico de Morse (1833) y estaba subordinada a la historia de las telecomunicaciones, siendo un paso más en dicha historia.

Por otro lado, por supuesto que nuestra economía es también una economía de producción. Pero en su mayor parte es una economía de servicios, de mercado y consumo, donde lo que se produce y mercantiliza como bienes más relevantes son productos financieros. Nuestros productos no nacen para hacer posible un futuro proyectado, ni dentro de una tarea histórica que nos demande; lo cual no tendría porqué ser un problema si hubiéramos sabido hacerle hueco a la teoría, quiero decir, si hubiéramos sabido separar la búsqueda teórica de la práctica económica. En realidad la filosofía es, frente a las ciencias, el ejercicio de esta desvinculación. Lo es desde sus inicios, y después de la crítica de la razón pura, la misma crítica de la razón práctica de Kant inicia una tarea por recuperar la autonomía del pensar que se extiende al idealismo alemán e incluso al origen de la fenomenología. Pero esta desvinculación no se ha logrado. Y la crisis actual de la filosofía en el fondo es el fracaso de la teoría para ejercerse independientemente de las necesidades económicas. Y como quiera que hoy la economía ya no es el ejercicio liberador de antaño que exigía la praxis teórica, hoy la crisis de la filosofía (y en otro tiempo no hubiera sido así), es la deriva sin destino (no sabiendo escuchar la pregunta que se

\footnotetext{
20 “Por supuesto la economía contemporánea es una economía de producción, pero esa economía de producción es una economía de servicios en más del 50 por 100. Por otra parte es una economía de mercado, pero esa economía de mercado es también una economía de consumo en el que el consumo mismo es también producción, y en la que, entre los bienes más relevantes, se mercantilizan y consumen productos financieros" J. Choza, Antropología filosófica. Las representaciones del sí mismo (Biblioteca Nueva, Madrid, 2008) p 169
} 
impone $^{21}$ ), que arrastra a la humanidad, y por eso la tarea del idealismo alemán (el último gran esfuerzo moderno por salvar la teoría) fue pensar la libertad como tarea.

\section{CODA}

Me atrevo a sugerir que sólo el homo que busca merece obtener el rango de la doble sabiduría, y dejar, por simpatía evolutiva, al hombre prehistórico el rango de sapiens a secas. Aunque no veo con malos ojos la propuesta de homo neciens defendida por Juan A. García en las I jornadas de la OMAU de Málaga. Si utilizamos el mismo rasero, homo neciens es cada vez más el hombre de la actualidad, mucho más cercano al hombre de Nearthertal que el hombre del Antiguo Egipto, Mesopotamia o América Central. Sin embargo, si tiene razón Kunt y la crisis es la condición primera y necesaria para el surgimiento de nuevas teorías, el final no debe ser trágico.

La extraña y crítica situación actual de las ciencias, desvinculada de su proyecto emancipador, había sido anunciada y pensada por Husserl antes incluso del declive hacia lo que Juan A. García ha sabido denominar como fobiasofía ${ }^{22}$. Lo que observó Husserl concretamente es el intento de reducir la ciencia a datos de hecho, reivindicando abiertamente que los objetos de la fenomenología son las esencias de los datos de hecho. Cuando el mundo académico registró la tesis de Husserl, entendió sencillamente que estábamos ante una nueva propuesta metodológica; que había aparecido una nueva etiqueta, incluso un nuevo modo de hacer filosofía después del idealismo. Esta nueva corriente se extendió rápidamente erigiéndose como el nuevo modo de hacer filosofía, aunque pronto tuvo que competir con otras formas nacientes de hacer filosofía a la que hemos dado el nombre de fobiasofía, y que se ha revelado finalmente como la extensión de aquella crisis abierta a la que quería hacer frente la fenomenología. Si hubiéramos observado que la crisis de la que hablaba Husserl era la crisis de la historia del pensar quizás hubiéramos estado prevenidos, pero no nos dimos cuenta, ni siquiera después de que Heidegger radicalizara las posiciones ante el escándalo de su maestro una vez que había advertido ya que dicha crisis era el destino del pensar.

Pero ni siquiera la radical postura heideggeriana tuvo éxito, se pensó sin más que se trataba de la tesis historicista según la cual toda filosofía pertenece a su propio tiempo, y ante este corto ángulo de mira, se escapó del horizonte de reflexión

\footnotetext{
${ }^{21}$ „Dichtung ist: das Dasein des Volkes in den Bereich dieser Weisung die Götter offenbar werden, nicht als irgendetwas Germeintes und Betrachtbares, sondern in ihrem Winken" M. Heidegger, Hölderlins Hymnen "Germanien" und "Der Rhein" (Klostermann, Frankfurt a.M., 1999) p 32

${ }_{22}$ "el pensamiento de la diferencia corre el peligro de mudarse en un rechazo visceral, o en una negación vital al margen del pensar. Nietzsche abre las puertas a esta posibilidad, de acuerdo con la cual nos abocamos a un estricto no saber, y a lo que habría que llamar fobiasofía" J. A. García González, Después de Husserl. Estudios sobre la filosofía del siglo XX (Librosenred, Buenos Aires, 2006) p 27
} 
el hecho de que Heidegger hablaba del peligro en el que se hallaba la meditación teórica ${ }^{23}$.

$* * * * *$

Alejandro Rojas Jiménez es miembro del grupo de investigación sobre el idealismo alemán: Sclleling (HUM-172) en la Universidad de Málaga.

Publicaciones recientes:

La libertad en la filosofía de la Cuadratura de Heidegger, en Thémata 41 (2009), pp 541547

La tarea de la filosofía, en Navarro Cordón-Vicente SanFélix (coords.) 2009: Pensar el futuro, SAF, Madrid, 2009, pp 1-10

Lebenswelt und Wissenschaft, en TABVLA RASA. Jenenser Zeitschrift für Kritisches Denken, Verlag Jena, in der Ausgabe 34 (IV/2008) 1-8

Líneas de investigación:

Recientemente ha defendido en la Universidad de Málaga su tesis doctoral sobre la Cuadratura heideggeriana con la calificación de sobresaliente cum laude por unanimidad

Dirección postal:

Universidad de Málaga. Departamento de filosofía. Facultad de filosofía y letras. Campus de Teatinos. 29071 Málaga (España)

Dirección electrónica:

rojas a@uma.es

proceso de selección del trabajo:

solicitado: 1 de enero de 2009

recibido: 14 de abril de 2009

aceptado: 14 de abril de 2009

${ }_{23}$ "Mu $\beta$ der Mensch aus der Tiefe des heimatlichen Bodens in den Äther hinaufsteigen können. Äther bedeutet hier: die freie Luft des hohen Himmels, den offenen Bereich des Geistes" M. Heidegger Gelassenheit cit, pp 14-15 